\title{
Electronic Structure of an XUV Photogenerated Solid-Density Aluminum Plasma
}

S. M. Vinko, ${ }^{1, *}$ U. Zastrau, ${ }^{2}$ S. Mazevet ${ }^{3}$ J. Andreasson, ${ }^{4}$ S. Bajt ${ }^{5}$ T. Burian, ${ }^{6}$ J. Chalupsky, ${ }^{6}$ H. N. Chapman, ${ }^{7,8}$ J. Cihelka, ${ }^{6}$ D. Doria, ${ }^{9}$ T. Döppner, ${ }^{10}$ S. Düsterer, ${ }^{5}$ T. Dzelzainis, ${ }^{9}$ R. R. Fäustlin, ${ }^{5}$ C. Fortmann, ${ }^{10}$ E. Förster,${ }^{2}$ E. Galtier ${ }^{11}$ S. H. Glenzer, ${ }^{10}$ S. Göde, ${ }^{12}$ G. Gregori,${ }^{1}$ J. Hajdu,${ }^{4}$ V. Hajkova, ${ }^{6}$ P. A. Heimann, ${ }^{13}$ R. Irsig, ${ }^{12}$ L. Juha, ${ }^{6}$ M. Jurek, ${ }^{14}$ J. Krzywinski, ${ }^{15}$ T. Laarmann, ${ }^{5}$ H. J. Lee, ${ }^{15}$ R. W. Lee, ${ }^{10}$ B. Li, ${ }^{1}$ K.-H. Meiwes-Broer, ${ }^{12}$ J. P. Mithen, ${ }^{1}$ B. Nagler, ${ }^{16}$ A. J. Nelson, ${ }^{10}$ A. Przystawik, ${ }^{12}$ R. Redmer, ${ }^{12}$ D. Riley,${ }^{9}$ F. Rosmej, ${ }^{11}$ R. Sobierajski, ${ }^{17,14}$ F. Tavella, ${ }^{5}$ R. Thiele, ${ }^{12}$ J. Tiggesbäumker, ${ }^{12}$ S. Toleikis, ${ }^{5}$ T. Tschentscher ${ }^{18}$ L. Vysin, ${ }^{6}$ T. J. Whitcher, ${ }^{1}$ S. White, ${ }^{9}$ and J. S. Wark ${ }^{1}$ ${ }^{1}$ Department of Physics, Clarendon Laboratory, University of Oxford, Parks Road, Oxford, OX1 3PU, United Kingdom ${ }^{2}$ Institut für Optik und Quantenelektronik, Friedrich-Schiller-Universität, Max-Wien-Platz, 1, 07743 Jena, Germany ${ }^{3} C E A, D A M, D I F, F-91297$ Arpajon, France

${ }^{4}$ Laboratory of Molecular Biophysics, Uppsala University, Box 596, SE-751 24, Uppsala, Sweden ${ }^{5}$ Deutsches Elektronen-Synchrotron DESY, Notkestrasse 85, D-22607 Hamburg, Germany ${ }^{6}$ Institute of Physics ASCR, Na Slovance 2, 18221 Prague 8, Czech Republic

${ }^{7}$ Center for Free-Electron Laser Science at DESY, Notkestrasse 85, D-22607 Hamburg, Germany

${ }^{8}$ University of Hamburg, Luruper Chaussee 149, D-22761 Hamburg, Germany

${ }^{9}$ Queen's University Belfast, University Road, Belfast, BT7 INN, Northern Ireland, United Kingdom

${ }^{10}$ Lawrence Livermore National Laboratory, 7000 East Avenue, Livermore, California 94550, USA

${ }^{11}$ University Pierre et Marie Curie, LULI, case 128, 4 place Jussieu, 75252 Paris Cedex 05, France

${ }^{12}$ Institut für Physik, Universität Rostock, D-18051 Rostock, Germany

${ }^{13}$ Lawrence Berkeley National Laboratory, 1 Cyclotron Road, Berkeley, California 94720, USA

${ }^{14}$ Institute of Physics PAS, Al. Lotnikw 32/46, PL-02-668 Warsaw, Poland

${ }^{15}$ SLAC National Accelerator Laboratory, 2575 Sand Hill Road, Menlo Park, California 94025, USA

${ }^{16}$ Photon Science Research Institute, Science and Technology Facilities Council, Didcot, United Kingdom

${ }^{17}$ FOM-Institute for Plasma Physics Rijnhuizen, NL-3430 BE Nieuwegein, The Netherlands

${ }^{18}$ European XFEL GmbH, Albert-Einstein-Ring 19, 22761 Hamburg, Germany

(Received 15 April 2010; published 1 June 2010)

By use of high intensity XUV radiation from the FLASH free-electron laser at DESY, we have created highly excited exotic states of matter in solid-density aluminum samples. The XUV intensity is sufficiently high to excite an inner-shell electron from a large fraction of the atoms in the focal region. We show that soft-x-ray emission spectroscopy measurements reveal the electronic temperature and density of this highly excited system immediately after the excitation pulse, with detailed calculations of the electronic structure, based on finite-temperature density functional theory, in good agreement with the experimental results.

DOI: 10.1103/PhysRevLett.104.225001

PACS numbers: 52.27.Gr, 71.15.Mb, 78.70.En

The development and availability of 4th generation light sources now provides the opportunity to study the interaction of XUV and $\mathrm{x}$-ray radiation with matter at intensities that have hitherto remained the province of optical lasers. The interaction of such radiation with solid-density conducting matter differs fundamentally from that of optical radiation, as the frequency of the XUV light is significantly higher than the plasma frequency of a solid-density metal. It has been proposed that, given the relatively long absorption lengths of such radiation, intense ultrashort 4th generation sources may provide a means to heat matter isochorically to temperatures from a few $\mathrm{eV}$ up to hundreds of $\mathrm{eV}$, thereby producing warm dense matter [1]. Many of the properties of such matter-e.g., the equation of state, transport properties, ion structure factor, and electronic density of states-are poorly understood: from the theoretical standpoint it is difficult to model a system where thermal energies and Coulomb interaction energies are of the same order, and from the experimental point of view the generation and subsequent diagnosis of samples free from gradients in temperature and density has remained elusive.

It is in this context that we report on recent experiments performed using the Free Electron Laser in Hamburg (FLASH at DESY) [2], operating at a $5 \mathrm{~Hz}$ repetition rate, with each pulse about 35 fs long [3] and a wavelength of $13.5 \mathrm{~nm}(92 \mathrm{eV})$, where the XUV radiation was focused onto solid $\mathrm{Al}$ targets at intensities up to $10^{16} \mathrm{~W} \mathrm{~cm}^{-2}$. The photon energy used is significantly higher than the plasma frequency of $\mathrm{Al}(15 \mathrm{eV})$ so that the laser penetration into the sample is not limited to the surface but results in volumetric photo absorption and consequent heating. As the valence band excitation cross section is small for photons at $92 \mathrm{eV}$ in $\mathrm{Al}$ [4], the dominant absorption channel is the excitation of one of the electrons in the $2 p$ core states, which give rise to the $L$ edge at $73 \mathrm{eV}$ [5]. This excitation reduces the screening of the remaining $2 p$ electrons and shifts the $L$ edge to $93.4 \mathrm{eV}$ [6], an energy greater 
than the XUV photon energy, thus rendering the photoexcited $\mathrm{Al}$ atom largely transparent to the rest of the pulse. This mechanism was recently used to demonstrate for the first time saturable absorption in the XUV, where the FLASH pulse was focused to intensities sufficiently high to excite every $\mathrm{Al}$ atom in the focal region of the target sample [7].

The $2 p$ core hole recombination is dominated by Auger decay, and has a lifetime of about $40 \mathrm{fs}$ [8]. Therefore, by the end of the excitation pulse the number of electrons in the valence band will be increased from three, in the cold solid, to a maximum of four at the highest intensities, when a single core electron is excited from every atom. As it takes several picoseconds for the electron energy to transfer to the lattice [9], we infer that the ion cores themselves remain close to their original positions, defined by the facecentered-cubic (fcc) lattice of the solid. At this stage in its evolution, the system is not in thermal equilibrium, as all of the absorbed XUV energy has been transferred to the electrons, while the ions are cold. However, the properties of this exotic state are of great interest, as the matter corresponds to a novel form of plasma of known density and of known ion-ion structure factor. In this letter we report on valence band to $L$-shell soft-x-ray emission spectroscopy (XES) measurements which provide information about the local electronic density of states (LDOS) of this system, and show how it alters as a function of the XUV intensity. Hence, we relate the XUV intensity to the degree of ionization of the plasma and the electron temperature.

The emission spectrum is dictated by the LDOS of the final state in the recombination process-the so-called final state rule [10]. At low irradiances, and thus low ionization fractions, when a core-hole is filled it is highly unlikely that any neighboring atom is in an excited state, and the emission will map the available partial DOS of the system in the ground state, where all of the ionic cores are $\mathrm{Al}^{3+}$. The average laser intensity above which an emitting atom will have a nearest neighbor in an excited state can be roughly estimated for a cubic system by $I_{\min }=$ $g E_{\mathrm{ph}} a_{0}^{-3} \tau^{-1} 3 \eta\left(E_{\mathrm{ph}}\right)$, where $g$ is the ratio between the number of atoms in the unit cell and the number of nearest neighbors, $E_{\mathrm{ph}}$ is the excitation photon energy, $a_{0}$ the lattice constant, $\tau$ the core hole recombination time, and $\eta$ the penetration depth within the sample. For bulk Al, $I_{\min }$ is of order $2 \times 10^{13} \mathrm{~W} \mathrm{~cm}^{-2}$, which is readily accessible in our experimental setup. Above this intensity the emitting atom has a non-negligible probability of having another excited $\left(\mathrm{Al}^{4+}\right.$ core) atom as its neighbor, and thus the relevant final state of the system-probed by the XESis in fact the LDOS of an $\mathrm{Al}^{3+}$ ion surrounded by neighbors that can be either $\mathrm{Al}^{3+}$ or $\mathrm{Al}^{4+}$, in a proportion that is a function of the XUV laser intensity.

In order to obtain data over a large range of intensities, two experimental setups were used and are illustrated in Fig. 1. In the first setup the energy of the pulse was varied using a gas attenuator, providing between $1-50 \mu \mathrm{J}$ on target, in a constant spot size of $20 \times 30 \mu \mathrm{m}^{2}$ [11]. This setup was used for the data collected at lower intensities. Higher intensities were achieved on the microfocusing beam line, where the FLASH beam was focused using a $\mathrm{Si} /$ Mo-multilayer-coated off-axis parabola [12]. In this setup the energy is kept approximately constant $(\sim 9 \mu \mathrm{J}$ on target) while the position of the target with respect to focus is varied producing effective spot sizes between 1-10 $\mu \mathrm{m}$. In this manner the emission spectrum was collected for targets subjected to irradiances ranging over 3 orders of magnitude. In both setups the total energy within the beam was monitored on a shot-to-shot basis by means of a gas monitor detector [13], from which the average pulse energy of each exposition was calculated.

The XES spectrum of the bulk Al target was recorded using the high-throughput XUV spectrometer HiTRaX, featuring a toroidal focusing mirror and a variable line space reflection grating, with a spectral range of 5-35 nm and a resolution of $\lambda / \Delta \lambda=330$ at $21 \mathrm{~nm}$ [14]. For each recorded spectrum the exposure time was between 10-60 s, depending on the pulse energy, during which the beam was delivered onto a continuously moving target to irradiate a fresh portion for each shot. An electron photoexcited into the valence band from the $2 p$ core state has an energy of about $20 \mathrm{eV}$ above the Fermi energy, and we assume it thermalizes on a time scale on the order of several fs [15]. The majority of electrons recombine via Auger decay and the radiative yield is only $0.24 \%$ [16]. The experimental spectra are shown as a function of on-target intensity in Fig. 2. We see noticeable change in the emission spectrum, which is associated with the changes in the electronic temperature and valence density with increasing XUV intensity.

To better understand this system and the changes it undergoes as the number of core holes increases, we have conducted first-principle calculations using finitetemperature density functional theory combined with molecular dynamics (QMD), as implemented in the ABINIT code $[17,18]$. For all the calculations presented here, we used 32 atoms in the simulation cell with a Brillouin zone

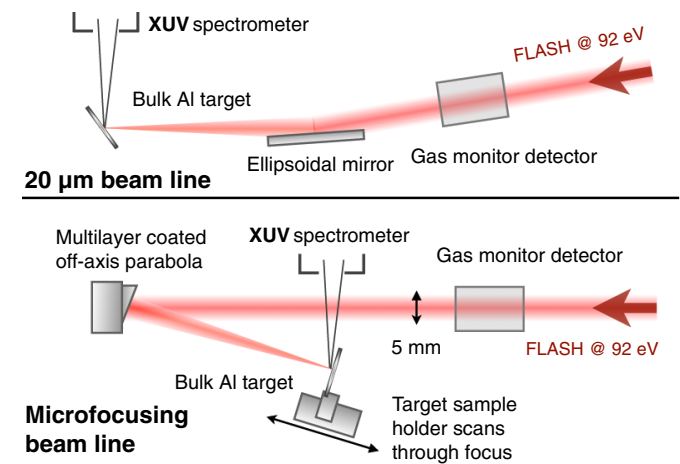

FIG. 1 (color online). Schematic view of the two experimental setups to access a range of intensities between $10^{13}-10^{16} \mathrm{~W} \mathrm{~cm}^{-2}$. 


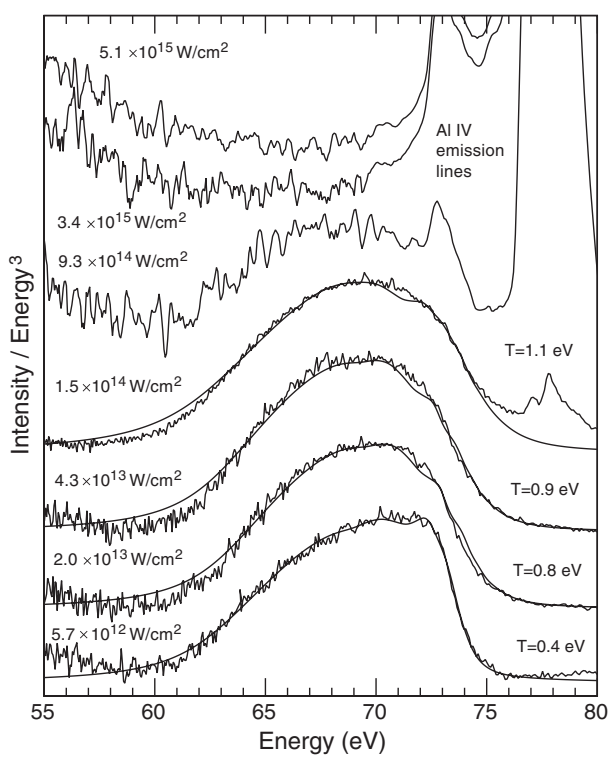

FIG. 2. Valence band to $2 p$ emission in solid-density $\mathrm{Al}$ for an increasing range of FEL irradiation intensities. QMD calculations are for a final state with $0,1,3$, and 10 core holes per 32atom supercell. Emission from atomic transitions in the $\mathrm{Al}$ plasma formed later in time as the target expands dominates the spectra at high intensities. Spectra are normalized to the same intensity at $72 \mathrm{eV}$ and offset for clarity.

sampling using a $10 \times 10 \times 10 \mathbf{k}$-point grid following the Monkhorst-Pack scheme [19]. The exchange and correlation potentials are in the Perdew, Burke, and Ernzerhof form of the generalized gradient approximation [20] and generated using the ATOMPAW code [21]. Following previous calculations performed for XANES spectra [22,23], we consider the system to be composed of two distinct types of atoms, which are represented using projector augmented wave potentials [24,25]. Ground state $\mathrm{Al}$ atoms are constructed with a frozen $\mathrm{Al}^{3+}$ core and the remaining three electrons in the valence band. The excited atoms are in turn given by a frozen $\mathrm{Al}^{4+}$ core with a single core hole in the $2 p$ state, and four valence electrons. The atoms are then randomly populated inside the 32-atom fcc lattice corresponding to a density of $2.7 \mathrm{~g} / \mathrm{cm}^{3}$. By comparing the core state energies of the two potentials we find the $L$-edge shift upon excitation to be $(-20.4 \pm 0.1) \mathrm{eV}$, a result in good agreement with measurements from Auger spectroscopy of $(-20.5 \pm 0.2) \mathrm{eV}[6]$. We have compared the $\mathrm{Al}^{4+}$ ion to a $\mathrm{Si}$ ion placed in an $\mathrm{Al} \mathrm{fcc}$ cell, as previously performed by Chetty and co-workers [26], and the electronic structure of the two are seen to be similar, in agreement with the equivalent-core approximation [27]. Further details on the calculations will be given in a following publication.

In the one-electron picture, the soft-X-ray emission radiated power due to transitions from the Al valence states $\left|\varphi_{n}\right\rangle$ to the inner core $2 p$ state $\left|\psi_{2 p}\right\rangle$ is given by

$$
I(E) \propto E^{2} \sum_{n, k}\left|\left\langle\psi_{2 p}\left|\nabla_{\mathbf{r}}\right| \varphi_{n k}\right\rangle\right|^{2} \delta\left(E-E_{2 p}+\varepsilon_{n k}\right),
$$

where $E_{2 p}$ is the energy level of the core state and $\varepsilon_{n}$ are the energy levels of the valence states. The summation is over all occupied bands $n$ and sampled $k$ points in the Brillouin zone. The one-electron picture neglects higherorder effects, such as intraband electron collisions and multielectron transitions and also broadening due to finite core-hole lifetimes. These effects lead to both a general broadening in the spectrum and to a low energy tail in the emission, which can be approximately described cohesively as a general, energy dependent level broadening. To compare experimental spectra with our calculations we use the one-parameter expression for the broadening proposed by Landsberg [28], determined empirically from low intensity data.

Assuming the excited electrons thermalize with the valence band before the emission takes place, we can extract the electron temperature from the spectra based on the slope on the high-energy edge of the emission peak, as shown in Fig. 2. An important caveat is that only a spatially and temporally averaged temperature can be extracted as the spectra are integrated over the entire emission time, during which the valence band heats due to the Auger recombination, and is emitted from all of the excited regions of the target. We find the temperatures to be about $0.4 \mathrm{eV}$ at low intensities $\left(\sim 10^{13} \mathrm{~W} \mathrm{~cm}^{-2}\right)$ and $1.1 \mathrm{eV}$ at medium intensities $\left(\sim 10^{14} \mathrm{~W} \mathrm{~cm}^{-2}\right)$, with an uncertainty below $0.1 \mathrm{eV}$, which are used as an input for the QMD calculations. These confirm that a change in electron temperature alone, up to several $\mathrm{eV}$, does not modify significantly the band structure of Al [29]. Such analysis cannot be performed at the highest laser intensities, due to appearance of intense plasma line emission overlapping the highenergy slope of the valence band fluorescence. These plasma lines, associated with atomic Al IV ions, are given by the emission from the much lower-density plasma formed upon expansion of the rapidly heated solid-density target. As electron-ion relaxation (and hence expansion) occurs on picosecond time scales [9], we infer this emission occurs significantly later than valence to core-hole emission at solid density.

The electron excitation due to the intense free electron laser (FEL) pulse dramatically changes the electron band structure, as can be seen in Fig. 3, where the calculated occupied LDOS around the two different ion cores, excited and nonexcited, is shown. This effect is spatially localized due to the efficient screening of the excess charge by the valence electrons, and as a result neighboring sites are weakly effected. The extent to which this holds is of considerable practical interest because perfect screening is often assumed in ion-in-cell plasma models, important for opacity and equation-of-state calculations [30,31], and can be directly estimated from XES from highly excited samples. We further note that at lower intensities the excited atom behaves like a $Z+1$ impurity, modifying the bottom of the band structure in a manner similar to that in $\mathrm{Mg}-\mathrm{Al}$ or Al-Si binary alloys [32]. 


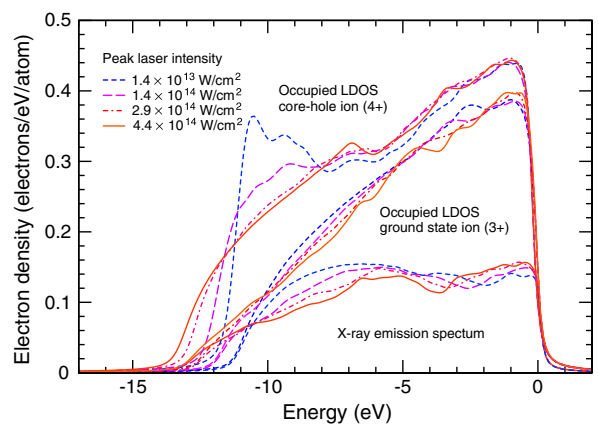

FIG. 3 (color online). Calculated local electron density within a sphere centered on a ground state ion and a core-hole excited ion. At low intensities the excited site is seen as an impurity and the bottom of the band is strongly modified. At high intensities the system returns to be free-electron-like but with an increased electron density. The one-electron contribution to the emission according to Eq. (1) is also shown.

For high XUV intensities, when more than half the atoms are excited, the band structure of the excited states returns to be very free-electron-like as in the case of ground state $\mathrm{Al}$, but now the number of valence electrons per atom is increased from three towards four. Such changes in the electronic structure are reflected in the XES in Fig. 3 through the LDOS and the transition matrix elements as given in Eq. (1). Interestingly, although the LDOS around the $\mathrm{Al}^{4+}$ ions is significantly altered as a function of intensity (and thus $\mathrm{Al}^{4+}$ to $\mathrm{Al}^{3+}$ ratio), the change in the LDOS around the $\mathrm{Al}^{3+}$ ions (which is what is measured experimentally, owing to the final state rule), while observable, is less pronounced. That said, the changes that are predicted by the calculations (a slight widening and broadening of the band), provide good agreement with the experimental data, as can be seen by the comparison between experiment and theory in Fig. 2.

In conclusion, we have performed XUV spectroscopy on highly excited samples of solid-density Al. XES measurements provide detailed information on the electronic temperature and valence density immediately after the excitation pulse. The valence band temperature is seen to increase up to $1.1 \mathrm{eV}$ which is the dominant effect to the emission spectrum as the intensity is increased up to moderate values. $A b$ initio calculations predict a dramatic change in the LDOS around the $\mathrm{Al}^{4+}$ ions as a function of intensity. While changes in the LDOS around the $\mathrm{Al}^{3+}$ ions (corresponding to the final state, and thus the emission spectrum) are less pronounced, they are consistent with the experimental spectra, providing confidence in our ability to model the electronic structure of warm dense matter.

The authors would like to acknowledge useful discussions with P. Weightman. Portions of this research were carried out at FLASH at HASYLAB/DESY. DESY is a member of the Helmholtz Association. The authors want to acknowledge support for access to FLASH by DESY and the European Community under Contract No. RII3-CT-
2004-506008 (IA-SFS), the German Federal Ministry for Education and Research via Project No. FSP 301-FLASH, the UK STFC and EPSRC Grant No. EP/G007187/1, the Oxford Supercomputing Centre, the Czech Ministry of Education (LC510, LC528, LA08024, and ME10046), Czech Science Foundation (202/08/H057), Czech Academy of Sciences (Z10100523, IAAX00100903, KAN300100702). This work was partially performed under the auspices of the U.S. Department of Energy by Lawrence Livermore National Laboratory, Contract No. DE-AC52-07NA27344. This work was further supported by Grant No. 08-ERI-002.

*sam.vinko@physics.ox.ac.uk

[1] R. W. Lee et al., J. Opt. Soc. Am. B 20, 770 (2003).

[2] W. Ackermann et al., Nat. Photon. 1, 336 (2007).

[3] U. Frühling et al., Nat. Photon. 3, 523 (2009).

[4] S. M. Vinko et al., High Energy Density Phys. 5, 124 (2009).

[5] B. L. Henke, E. M. Gullikson, and J.C. Davis, At. Data Nucl. Data Tables 54, 181 (1993).

[6] G. Dufour et al., Phys. Scr. 13, 370 (1976).

[7] B. Nagler et al., Nature Phys. 5, 693 (2009).

[8] C. O. Ambladh et al., Phys. Rev. B 39, 3489 (1989).

[9] B. J. Siwick et al., Science 302, 1382 (2003).

[10] U. von Barth and G. Grossmann, Phys. Rev. B 25, 5150 (1982).

[11] U. Zastrau et al., Phys. Rev. E 78, 066406 (2008).

[12] A. J. Nelson et al., Opt. Express 17, 18271 (2009).

[13] K. Tiedtke et al., New J. Phys. 11, 023029 (2009).

[14] R. R. Fäustlin et al., JINST 5, P02004 (2010).

[15] I. Campillo, J. M. Pitarke, and A. G. Eguiluz, Phys. Rev. B 58, 10307 (1998).

[16] W. Bambynek et al., Rev. Mod. Phys. 44, 716 (1972).

[17] X. Gonze et al., Comput. Mater. Sci. 25, 478 (2002).

[18] N. D. Mermin, Phys. Rev. 137, A1441 (1965).

[19] H. Monkhorst and J. Pack, Phys. Rev. B13, 5188 (1976).

[20] J. Perdew, K. Burke, and M. Ernzerhof, Phys. Rev. Lett. 77, 3865 (1996).

[21] N. Holzwarth, Comput. Phys. Commun. 135, 329 (2001).

[22] S. Mazevet and G. Zérah, Phys. Rev. Lett. 101, 155001 (2008).

[23] V. Recoules and S. Mazevet, Phys. Rev. B80, 064110 (2009).

[24] P. E. Blöchl, Phys. Rev. B 50, 17953 (1994).

[25] M. Torrent et al., Comput. Mater. Sci. 42, 337 (2008).

[26] N. Chetty et al., Phys. Rev. B 52, 6313 (1995).

[27] W. L. Jolly and D. N. Hendrickson, J. Am. Chem. Soc. 92 , 1863 (1970).

[28] P. T. Landsberg, Proc. Phys. Soc. London 62, 806 (1949).

[29] V. Recoules et al., Phys. Rev. Lett. 96, 055503 (2006).

[30] T. Blenski, A. Grimaldi, and E. Perrot, Phys. Rev. E 55, R4889 (1997).

[31] T. Blenski and B. Cichocki, High Energy Density Phys. 3, 34 (2007).

[32] C. H. Zhang et al., Phys. Rev. B 37, 2401 (1988). 